\title{
Enhancement of stability in systems with metastable states
}

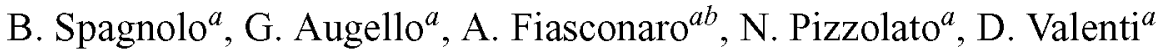 \\ ${ }^{a}$ Dipartimento di Fisica e Tecnologie Relative, Università di Palermo and \\ CNISM-INFM, Unità di Palermo, Group of Interdisciplinary Physics $\sharp$, \\ Viale delle Scienze, edificio 18, I-90128 Palermo, Italy \\ URL: hitp://gip.dft.unipa.it, e-mail address: spagnolo@unipa.it \\ ${ }^{b}$ Mark Kac Complex Systems Research Center, Institute of Physics, Jagellonian University, \\ Reymonta 4, 30-059 Kraków, Poland
}

\begin{abstract}
.
The investigation of noise-induced phenomena in far from equilibrium systems is one of the approach used to understand the behaviour of physical and biological complex systems. Metastability is a generic feature of many nonlinear systems, and the problem of the lifetime of metastable states involves fundamental aspects of nonequilibrium statistical mechanics. The enhancement of the lifetime of metastable states through the noise enhanced stability effect and the role played by the resonant activation phenomenon will be discussed in models of interdisciplinary physics: (i) Ising model (ii) Josephson junction; (iii) stochastic FitzHugh-Nagumo model; (iv) a population dynamics model, and (v) a market model with stochastic volatility.
\end{abstract}

Keywords: Metastability, noise enhanced stability, stochastic dynamics, stochastic modeling of biological and medical physies.

PACS: 05.10.Gg, 05.40.-a, 85.25.Cp, 87.23.Cc, 87.10.Mn, 87.19.11, 89.65.Gh

\section{INTRODUCTION}

Stabilization and delay of instability of dynamical systems using deterministic or stochastic external driving forces has been the focus of many studies by physicists, mathematicians and also oncologists $[1,2]$. The idea of stabilizing an inverted pendulum by imposing parametric excitation can be traced back to the work of Ref. [3]. In nonlinear dynamical systems, noise can shift the bifurcation threshold, or induce new phase transitions in spatially extended systems, or create spatial patterns [4]. Noise induced transitions implies a change in the number of extrema in the probability density function of the system response and near the transition a marked increase in the relaxation time occurs for cubic and for strongly asymmetric bistable systems [5].

For additive noise a physical quantity which characterize the stability of a metastable state is the mean first passage time or the relaxation time. This quantity gives a measure of the lifetime of the metastable state and it is strongly dependent on the potential profile of the physical system investigated as well as on the initial conditions and on the intensity of the additive random fluctuations or thermal fluctuations. The thermal activated escape from a metastable state is a ubiquitous problem in physics, chemistry, and biology [6]. The dependence of the lifetime or the relaxation time on the noise intensity for metastable and unstable systems was revealed to have resonance character. Typical examples are the resonant activation (RA) phenomenon [7]-[15], whose signature is a 
minimum of the lifetime of the metastable state as a function of a driving frequency, and the noise enhanced stability (NES) [10]-[19]. This resonance-like phenomenon, which contradicts the monotonic behavior predicted by the Kramers theory [20], shows that the noise can modify the stability of the system in a counterintuitive way, such that the system remains in the metastable state for a longer time than in the deterministic case and the escape time, or lifetime of the metastable state, has a maximum at some noise intensity [16]. When a Brownian particle is moving in a potential profile with a fluctuating metastable state, the NES effect is characterized by two different dynamical regimes: (a) a monotonic behavior with a divergence of the lifetime of the metastable state when the noise intensity tends to zero, for a particular range of unstable initial conditions (see for details Ref. $[16,17]$ ), which means that the Brownian particle will be trapped into the metastable state in the limit of very small noise intensities; (b) a nonmonotonic behavior of the lifetime of the metastable state as a function of the noise intensity and as a function of the driving frequency of the fluctuating potential (see Ref. [10]). Noise-enhanced stabilization out of equilibrium in a study of metastability and nucleation in a kinetic two-dimensional Ising model was recently predicted in Ref. [18]. Moreover a nonmonotonic behavior of the mean first passage time (MFPT) of a Brownian colloidal particle in a symmetric fluctuating periodic spatial potential as a function of the oscillation period was revealed [21]. The Brownian particle is moving in a channel geometry and the behavior of the MFPT is characterized by a minimum, that is a RA phenomenon, and a maximum which should be related to a signature of an enhancement of the stability of the metastable states of the fluctuating potential profile.

Relaxation in many natural proceeds through metastable states. This is often observed in condensed matter physics and also in various other fields, from cosmology to biology and high-energy physics. In spite of such ubiquity, the microscopic understanding of metastability still raises fundamental questions. This is because we are dealing with a typical out of equilibrium statistical dynamical phenomenon, not included in the ensemble formalism. One of the fundamental question is therefore related to the fluctuationdissipation theorem in transient dynamics with a metastable state which decays, triggered by rare random fluctuations, towards a non stationary state. In this work we will shortly review some recent results on thermal escape from metastable states in different physical systems by focusing on the enhancement of the lifetime of metastable states through the noise enhanced stability effect and the role played by the resonant activation phenomenon. Moreover, we will discuss these noise induced effects in models of interdisciplinary physics: (i) a kinetic Ising model; (ii) Josephson junctions; (iii) a stochastic FitzHugh-Nagumo model; (iv) a population dynamics model, and (v) a market model with stochastic volatility.

\section{THE MODEL}

Systems with a metastable state and strongly coupled with the noisy environment can be described by the following stochastic differential equation

$$
\frac{d x}{d t}=-\frac{\partial[U(x)+V(x) \eta(t)]}{\partial x}+\xi(t)
$$




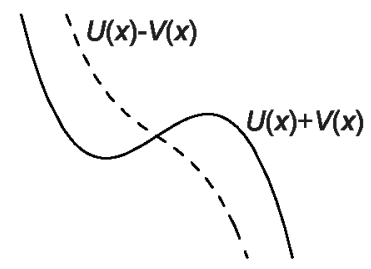

FIGURE 1. Switching potential with metastable state.

where $x(t)$ is the displacement of the Brownian particle and $\xi(t)$ is the white Gaussian noise with the usual statistical properties: $\langle\xi(t)\rangle=0,\left\langle\xi(t) \xi\left(t^{\prime}\right)\right\rangle=2 D \delta\left(t-t^{\prime}\right)$. The variable $\eta(t)$ is the Markovian dichotomous noise, which takes the values \pm 1 with the mean flipping rate $v$. The potential profile $U(x)+V(x)$ corresponds to a metastable state, and $U(x)-V(x)$ corresponds to an unstable one, with a reflecting boundary at $x \rightarrow-\infty$ and an absorbing boundary at $x \rightarrow+\infty$ (see Fig. 1). Starting from the wellknown expression for the probability density $P(x, t)=\langle\delta(x-x(t))\rangle$, using the auxiliary function $Q(x, t)=\langle\eta(t) \delta(x-x(t))\rangle$, and the Eq. (1), we obtain

$$
\begin{aligned}
& \frac{\partial P(x, t)}{\partial t}=\frac{\partial}{\partial x}\left[U^{\prime}(x) P+V^{\prime}(x) Q\right]+D \frac{\partial^{2} P}{\partial x^{2}}, \\
& \frac{\partial Q(x, t)}{\partial t}=-2 v Q+\frac{\partial}{\partial x}\left[U^{\prime}(x) Q+V^{\prime}(x) P\right]+D \frac{\partial^{2} Q}{\partial x^{2}} .
\end{aligned}
$$

The average lifetime of Brownian particles in the interval $\left(L_{1}, L_{2}\right)$, with the initial conditions $P(x, 0)=\delta\left(x-x_{0}\right)$ and $Q(x, 0)= \pm \delta\left(x-x_{0}\right)$, is

$$
\tau\left(x_{0}\right)=\int_{0}^{\infty} d t \int_{L_{1}}^{L_{2}} P\left(x, t \mid x_{0}, 0\right) d x .
$$

From the general equations (2) it is possible to calculate the average lifetime for potential profiles with metastable states [10]. We have two mean lifetimes $\tau_{+}\left(x_{0}\right)$ and $\tau_{-}\left(x_{0}\right)$, depending on the initial configuration of the randomly switching potential profile: $U(x)+V(x)$ or $U(x)-V(x)$ (see Fig. 1). By considering piece-wise linear potential profile with $U(x)=\infty$ for $x<0, U(x)=0$ for $0 \leq x \leq L, U(x)=k(L-x)$ for $x>L$, and $V(x)=a x(x>0,0<a<k)$, with $L_{1}=0$ and $L_{2}=b$ the boundary, we obtain the exact mean lifetime

$$
\tau_{-}\left(x_{i n}=0\right)=\frac{b}{k}+\frac{v L^{2}}{\Gamma^{2}}+\frac{a}{2 v \Gamma^{4}} f(D, \Gamma, v),
$$

where $\Gamma=\sqrt{a^{2}+2 v D}$. Here $f(D, \Gamma, v)$, which is also a function of the potential parameters $a, b, L, k$, has a complicated expression in terms of parameters $D, \Gamma$ and $v[10]$. It is worthwhile to note that Eq. (4) was derived without any assumptions on the white noise intensity $D$ and on the mean rate of flippings $v$ of the potential. In Fig. 2 we show the plots of the normalized mean lifetime $\tau_{-}\left(x_{i n}=0\right) / \tau_{0}$, Eq. (4), as a function of: (a) the noise intensity $D$ for fixed mean flipping rate, (b) the switchings mean rate $v$ of the potential profile. The maximum value of the average lifetime and the range of noise intensity values, where NES effect occurs, increases when $\omega$ decreases (see Fig. 2a). In Fig. $2 \mathrm{~b}$ we see that the average escape time exhibits a minimum at a resonant fluctuation 

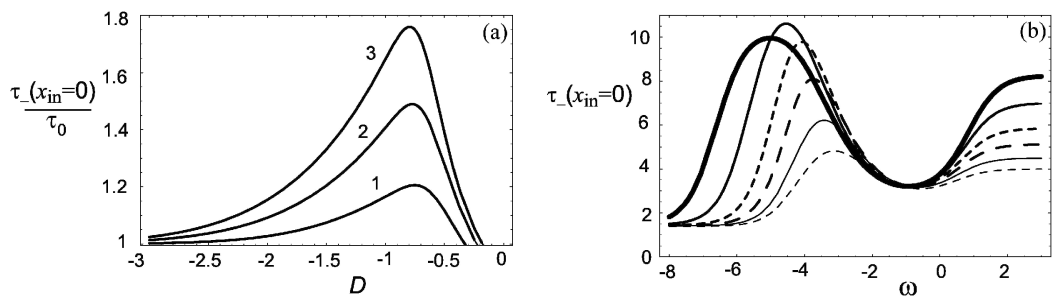

FIGURE 2. (a) Semilogarithmic plot of the normalized mean lifetime $\tau_{-}\left(x_{i n}=0\right) / \tau_{0}$ vs the white noise intensity $D$ for three values of the dimensionless mean flipping rate $\omega=v L / k: 0.03$ (curve 1), 0.01 (curve 2), 0.005 (curve 3). Parameters are $L=1, k=1, b=2$, and $a=0.995$. (b) Semilogarithmic plot of the mean lifetime $\tau_{-}(0)$ vs the dimensionless mean flipping rate $\omega=v L / k$ for six noise intensity values.

rate, that is the signature of the resonant activation (RA) phenomenon [7]. Moreover, in the same figure (b) a new resonance-like behaviour, is observed. The mean lifetime of the metastable state $\tau_{-}\left(x_{i n}=0\right)$ exhibits a maximum, between the slow limit of potential fluctuations (static limit) and the RA minimum, as a function of the mean fluctuation rate of the potential, . This maximum occurs for a value of the barrier fluctuation rate on the order of the inverse of the time $\tau_{u p}(D)$ required to escape from the metastable fixed configuration. This suggests that, the enhancement of stability of metastable state is strongly correlated with the potential fluctuations, when the Brownian particle "sees" the barrier of the metastable state [16]. When the average time to cross the barrier, that is the average lifetime of the metastable state, is approximately equal to the correlation time of the fluctuations of the potential barrier, a resonance-like phenomenon occurs. In other words, this new effect can be considered as a NES effect in the frequency domain. It is worthwhile to note that the new nonmonotonic behaviour shown in Fig. $2 b$ is in good agreement with experimental results observed in a periodically driven Josephson junction (JJ) [14]. In this very recent paper the authors experimentally observe the coexistence of RA and NES phenomena.

\section{NOISE ENHANCED STABILITY AND RESONANT ACTIVATION IN INTERDISCIPLINARY PHYSICS MODELS}

\section{Ising model}

The Ising model has been used as a prototype for the metastable dynamics of shortrange systems using Monte Carlo simulations in both two and three dimensions (see Ref. [18]). It was demonstrated that the metastable lifetimes for impurity-free kinetic Ising models exhibit system-size and field dependence. Hurtado et al. studied the simplest non-equilibrium Ising model with dynamic impurities and the interfaces that separate non-equilibrium phases. They predicted noise-enhanced stabilization of the nonequilibrium metastable states at low temperature (see Fig. 3). This is caused by the nonlinear interplay between thermal and non-equilibrium fluctuations, which induces anomalous, non-monotonous dependence on the temperature of the surface tension. The analytical modeling of the Ising model was based on a two-dimensional square lattice 


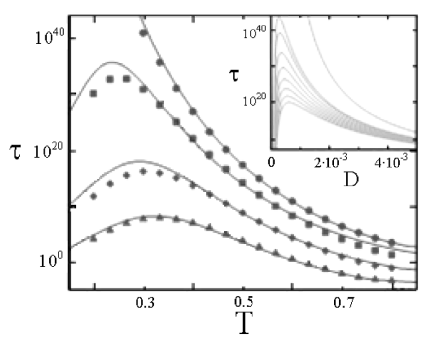

FIGURE 3. Lifetime $\tau$ as a function of normalized temperature $\mathrm{T}$ for the kinetic two-dimensional Ising model. Solid lines are theoretical predictions, while symbols represent Monte Carlo simulations. Inset: $\tau$ versus $D$ as derived from Langevin equation (5), with $h=-0.1$ and $\mu$ increasing from top to bottom [18].

with periodic boundary conditions and a spin variable at each node. Two different heat paths were found to compete in the model. Both induce completely random spin-flips, one with probability $p$, and the other with probability $(1-p)$. This competition resulted in non-equilibrium steady state sets. The strength of fluctuations affecting a spin was found to increase with the local order (the number of neighbors with the same state). This is typical of a multiplicative noise, and a Langevin equation with such a noise can capture the essential physics of the model

$$
\frac{\partial \psi}{\partial t}=\psi-\psi^{3}+h+\sqrt{D+\mu \psi^{2}} \xi(t)
$$

where $\psi$ is the transition rate of spin-flip, $h$ is the external magnetic field, $\xi(t)$ is a Gaussian white noise $\delta$ correlated, $D$ is the strength of the noise, and $\mu$ is the renormalized version of $p$. The Eq. (5) describes a Brownian particle moving in the effective potential

$$
V(\psi)=\psi^{2}-(1+D / \mu) \ln \left(\mu^{2}+D / \mu\right),
$$

obtained by the solution of the corresponding stationary Fokker-Planck equation, namely $p_{s t}(\psi)=A \exp [-V(\psi) / \mu]$ with A a normalization constant. The extrema of the effective potential $V(\mu)$, for $h<0$, correspond to metastable, stable, and unstable states. The above mean-field equation (5) contains the essential competition between thermal $(D)$ and nonequilirium $(\mu)$ fluctuations in a metastable potential that characterizes the physical system. This modeling was shown to recover the thermal NES phenomenon for the escape time observed in the microscopic model, that is the kinetic Ising model (see Fig. 3).

\section{Josephson junction}

The interplay of the noise-induced phenomena RA and NES on the temporal characteristics of the Josephson devices, and the role played by these noise-induced effects in the accumulation of timing errors in RSFQ logic devices, is analyzed in this section [15]. A small junction can be modelled by a resistance $\mathrm{R}$ in parallel with a capacitance $\mathrm{C}$, across which is connected a bias generator and a phase-dependent current 

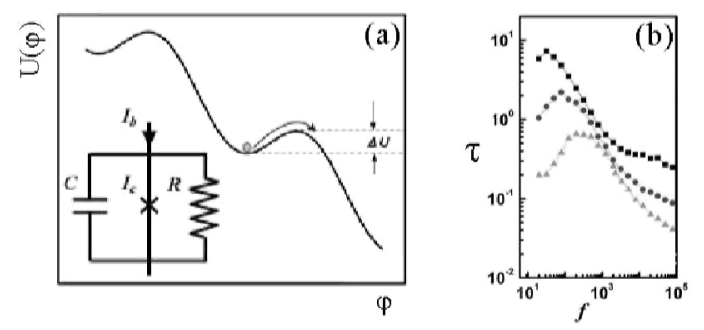

FIGURE 4. (a) Washboard potential and equivalent circuit of a current biased Josephson tunnel junction; (b) Average escape time as a function of the driving frequency for different bias currents. The resonant peaks indicate NES in the underdamped JJ sytem investigated [14].

generator, $I \sin \varphi$, representing the Josephson supercurrent due to the Cooper pairs tunnelling through the junction (see Fig. 4). Since the junction operates at a temperature above absolute zero, there will be a white Gaussian noise current superimposed on the bias current. Therefore the dynamics of a short overdamped JJ, widely used in logic elements with high-speed switching and corresponding to a negligible capacitance $C$, is obtained from the Josephson equation [11] and from the current continuity equation of the equivalent circuit of the Josephson junction. The resulting equation is the following Langevin equation

$$
\omega_{c}^{-1} \frac{d \varphi(t)}{d t}=-\frac{d u(\varphi)}{d \varphi}-i_{F}(t),
$$

valid for $\beta \ll 1$, with $\beta=2 e I_{c} R^{2} C / \hbar$ the McCamber-Stewart parameter, $I_{c}$ the critical current, and $i_{F}(t)=I_{F} / I_{c}$, with $I_{F}$ the random component of the current. Here $u(\varphi, t)=$ $1-\cos \varphi-i(t)$, with $i(t)=i_{0}+A \sin (\omega t)$, is the dimensionless potential profile (see Fig. $4 \mathrm{a}), \varphi$ is the difference in the phases of the order parameter on opposite sides of the junction, $i=I / I_{C}, \omega_{c}=\left(2 e R_{N} I_{c}\right) \hbar$ is the characteristic frequency of the $\mathrm{JJ}$, and $R_{N}$ is the normal state resistance (see Ref. [15]). The Gaussian noise current is characterized by: $\left\langle i_{F}(t)\right\rangle=0,\left\langle i_{F}(t) i_{F}(t+\tau)\right\rangle=\left(2 D / \omega_{c}\right) \delta(\tau)$, where $D=(2 e k T) /\left(\hbar I_{c}\right)=$ $I_{T} / I_{c}$ is the dimensionless intensity of fluctuations, $T$ is the temperature and $k$ is the Boltzmann constant. The equation of motion Eq. (7) describes the overdamped motion of a Brownian particle moving in a washboard potential (see Fig. 4a). A junction initially trapped in a zero-voltage state, with the particle localized in one of the potential wells, can escape out of the potential well by thermal fluctuations. The phase difference $\varphi$ fluctuates around the equilibrium positions, minima of the potential $u(\varphi)$, and randomly performs jumps of $2 \pi$ across the potential barrier towards a neighbor potential minimum. The resulting time phase variation produces a nonzero voltage across the junction with marked spikes. For a bias current less than the critical current $I_{\mathcal{C}}$, these metastable states correspond to "superconductive" states of the JJ. The mean time between two sequential jumps is the life time of the superconductive metastable state. For an external current greater than $I_{c}$, the JJ junction switches from the superconductive state to the resistive one and the phase difference slides down in the potential profile, which now has not equilibrium steady states. A Josephson voltage output will be generated in a later time. Such a time is the switching time, which is a random quantity. In the 

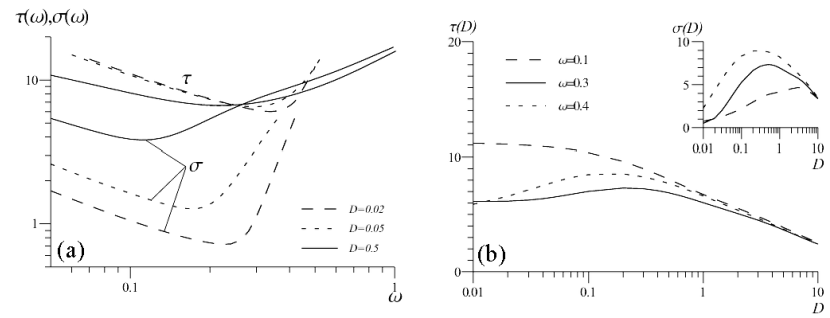

FIGURE 5. Theoretical results in an overdamped JJ system [11]: (a) The MST vs frequency for three values of the noise intensity; (b) The MST vs noise intensity for three values of the driving frequency. Inset: The standard deviation (SD) vs noise intensity for the same values of driving frequency $\omega$.

presence of thermal noise a Josephson voltage appears even if the current is less than the critical one $(i<1)$, therefore we can identify the lifetime of the metastable states with the mean switching time. Depending on the values of $i_{o}$ and $A$, as well as values of signal frequency and noise intensity, two noise-induced effects may be observed, namely the resonant activation (RA) and the noise enhanced stability (NES). Specifically the RA effect was theoretically predicted in Ref. $[7,11]$ and experimentally observed in a tunnel diode [8] and in underdamped Josephson tunnel junctions [9, 14], and the NES effect was theoretically predicted in $[10,11,16]$ and experimentally observed in a tunnel diode [16] and in an underdamped Josephson junction [14]. The RA and NES effects, however, have different role on the behavior of the temporal characteristics of the Josephson junction. Specifically, the RA phenomenon minimizes the switching time and therefore also the timing errors in RSFQ logic devices, while the NES phenomenon increases the mean switching time producing a negative effect. It is interesting to see that near the minimum (see Fig. 5a) the MST has a very weak dependence on the noise intensity, i. e. in this signal frequency range the noise is effectively suppressed [11]. We observe also the NES phenomenon. There is a frequency range in Fig. 5b where the switching time increases with the noise intensity. To see in more detail this effect we report in Fig. 5b the MST $\tau(D)$ and its $\operatorname{SD} \sigma(D)$ vs the noise intensity $D$, for three fixed values of the driving frequency. Both quantities have nonmonotonic behaviour.

\section{FitzHugh-Nagumo model}

The analysis of the stochastic properties of neural systems is of particular importance since it plays an important role in signal transmission. Here we analyze the effect of noise in a single neuron subjected to a strong periodic forcing. We investigate therefore, the influence of noise on the appearance time of a first spike, or the mean response time, in the output of FHN model with periodical driving in suprathreshold regime. We analyze the dependencies of the mean response time (MRT) on both driving frequency and noise intensity. The dynamics of the stochastic FitzHugh-Nagumo model can be expressed by two models: 

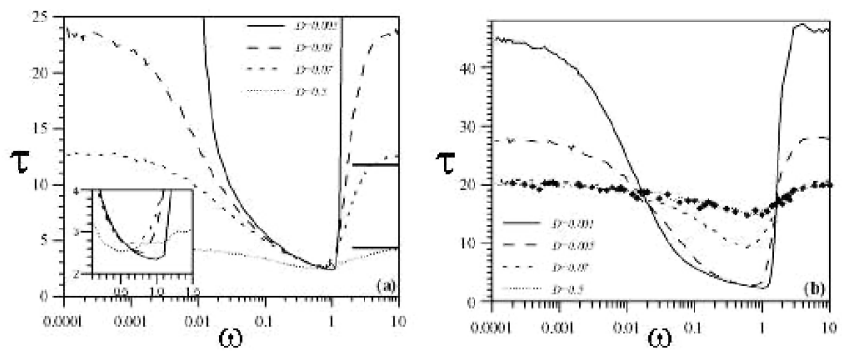

FIGURE 6. (a) The MRT vs the frequency of periodic driving for case $I$, for four values of the noise intensity. The right solid lines give the theoretical values of $\tau$ for fixed bistable potential. Inset: frequency range where the NES effect is observed. (b) The MRT vs the frequency of the periodic driving for case $I I$, for four values of the noise intensity. The curve with diamonds gives the values of $\tau$ for fixed bistable potential, when the noise source is a Wiener process.

Case $I$ The variable $x(t)$ that corresponds to the membrane potential is subjected to fluctuations, so we have

$$
\begin{aligned}
\dot{x}(t) & =x(t)-x^{3}(t) / 3+A \sin (\omega t)-y(t)+\xi(t) \\
\dot{y}(t) & =\varepsilon[x(t)+I],
\end{aligned}
$$

where $y(t)$ is the recovery variable, and $\varepsilon$ is a fixed small parameter $(\varepsilon=0.05)$. In the absence of both external driving and noise, there is only one steady state of the system (8), that is $x_{0}=-I ; y_{0}=-I+I^{3} / 3$.

Case $I I$ The recovery variable $y(t)$ associated with the refractory properties of a neuron is noisy, therefore we have

$$
\begin{aligned}
& \dot{x}(t)=x(t)-x^{3}(t) / 3+A \sin (\omega t)-y(t) \\
& \dot{y}(t)=\varepsilon[x(t)+I]+\xi(t),
\end{aligned}
$$

In Eqs. (8) and (9), $\xi(t)$ is a Gaussian white noise with zero mean and correlation function $\langle\xi(t) \xi(t+\tau)\rangle=D \delta(\tau)$. We observe a resonant activation-like phenomenon in both cases. The MRT exhibits a minimum as a function of the driving frequency, which is almost independent of the noise intensity (see Fig. 6). In a narrow frequency range $(\omega \in(0.6 \div 1.3)$ ) (see Fig. 6a), we found a nonmonotonic behavior of the MRT as a function of the noise intensity. Here the noise enhanced stability effect is observed (see the inset of Fig. 6a). Out of this range the MRT monotonically decreases with increasing noise intensity. For larger noise intensities the MRT dependence on driving frequency takes a constant-like behavior in the range of the investigated frequency values. Here, the dynamics of the system is mainly controlled by the noise, and the frequency of periodic driving does not affect significantly the neuron response dynamics. By numerical simulations of our system we find that, for large noise intensities, the MRT coincides with that calculated by standard technique for a Brownian particle moving in a bistable fixed potential. The theoretical values reported in Fig. 6(a) agree with the limiting values of $T$ for $\omega \rightarrow 0$ and $\omega \rightarrow \infty$. For $\varepsilon \ll 1$ in fact, $x(t)$ is a fast variable and $y(t)$ is a slow variable, so $\dot{y}(t) \simeq 0$ and this case can be recast as an escape problem from a one-dimensional double well in both limiting cases. In fact when $\omega \rightarrow 0$ we have a 


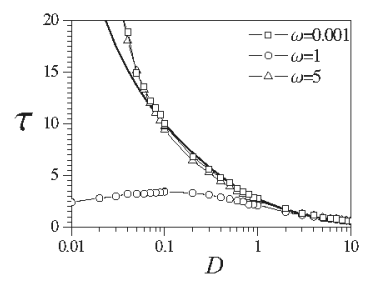

FIGURE 7. The mean response time dependence versus the noise intensity for case $I$, for three different values of driving frequency: $\omega=0.001, \omega=1$ and $\omega=5$. Solid line gives the theoretical values of $\tau$ for fixed bistable potential.

fixed bistable potential, and for $\omega \rightarrow \infty$ we have an average fluctuating potential, which coincides with the fixed one. This is well visible in Fig. 6(a) for $D=0.07$. For $D=0.5$ the MRT tends to be almost independent on the parameter $\omega$.

In Fig. 7 the MRT versus the noise intensity, for three values of the driving frequency, is shown. We see the nonmonotonic behavior for $\omega=1$, which is a signature of the NES effect. It is interesting to note that even in this system, whose global dynamics cannot be described as the motion of a Brownian particle in a potential profile (because of the coupling between the two stochastic differential equations describing our system) a phase transition-like phenomenon, with respect to the driving frequency parameter $\omega$, occurs. In fact we have nonmonotonic and monotonic behavior depending on the value of $\omega$. We expect similar behavior, if we fix the driving frequency and we change the value of the amplitude $A$ of the driving force [10].

\section{Cancer growth dynamics}

In this section we shortly summarize some of the main results obtained with a stochastic model for cancer growth dynamics (see Ref. [13]). Most of tumoral cells bear antigens which are recognized as strange by the immune system. A response against these antigens may be mediated either by immune cells such as T-lymphocytes or other cells like macrophages. The process of damage to tumor proceeds via infiltration of the latter by the specialized cells, which subsequently develop a cytotoxic activity against the cancer cell-population. The series of cytotoxic reactions between the cytotoxic cells and the tumor tissue have been documented to be well approximated by a saturating, enzymatic-like process whose time evolution equations are similar to the standard Michaelis-Menten kinetics. The T-helper lymphocytes and macrophages, can secrete cytokines in response to stimuli. The functions that cytokines induce can both "turn on" and "turn off" particular immune response. This "on-off" modulating regulatory role of the cytokines is here modelled through a dichotomous random variation of the parameter $\beta$, which is responsibile for regulatory inhibition of the population growth, by taking into account the natural random fluctuations always present in biological complex systems. The dynamical equation of this biological system is 


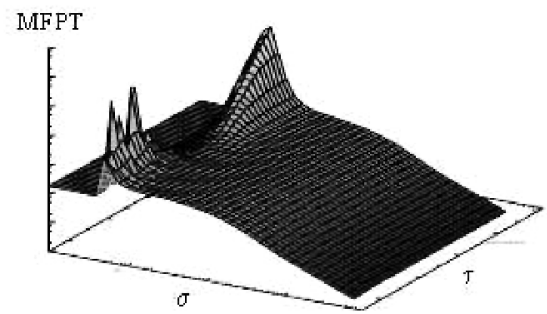

FIGURE 8. The coexistence of NES and RA phenomena in a three-dimensional plot of MFPT as a function of noise intensity $\sigma$ and correlation time $\tau$.

$$
\dot{x}=-\frac{d U^{ \pm}(x)}{d x}+\xi(t)
$$

where $\xi(t)$ is a $\delta$-correlated Gaussian process with zero mean and $U^{ \pm}(x)=-x^{2} / 2+$ $\left(\theta x^{3}\right) / 3+\left(\beta_{o} \pm \Delta\right)(x-\ln (x+1))$ is the stochastic double well Michaelis-Menten potential with one the minima at $x=0$. Here $x(t)$ is the concentration of the cancer cells. The process $\beta=\left(\beta_{o} \pm \Delta\right)$ can change the relative stability of the metastable state of the potential profile. We note that the RA and NES phenomena act counter to each other in the cancer growth dynamics: the NES effect increases in an unavoidable way the average lifetime of the metastable state (associated to a fixed-size tumor state), while the RA phenomenon minimizes this lifetime. Therefore it is crucial to find the optimal range of parameters in which the positive role of resonant activation phenomenon, with respect to the cancer extinction, prevails over the negative role of NES, which enhances the stability of the tumoral state. These are just the main results of the paper [13], that is both NES and RA phenomena are revealed in a biological system with a metastable state, with a co-occurrence region of these effects (see Fig. 8). In this coexistence region the NES effect, which enhances the stability of the tumoral state, becomes strongly reduced by the RA mechanism, which enhances the cancer extinction.

\section{The modified Heston model}

In this last section we present a generalization of the Heston model recently proposed in Ref. [19], by considering a cubic nonlinearity instead of a linear term as in the original Heston model. This generalization represents a fictitious "Brownian particle" moving in an effective potential with a metastable state, in order to model those systems with two different dynamical regimes like financial markets in normal activity and extreme days. The equations of the new model are

$$
\begin{aligned}
& d x(t)=-\left(\frac{\partial U}{\partial x}+\frac{v(t)}{2}\right) d t+\sqrt{v(t)} d W_{1}(t) \\
& d v(t)=a(b-v(t)) d t+c \sqrt{v(t)} d W_{2}(t),
\end{aligned}
$$

where $U(x)=2 x^{3}+3 x^{2}$ is the effective cubic potential with a metastable state at $x_{m e}=0$, a maximum at $x_{M}=-1$, and a cross point between the potential and the $x$ axes at $x_{I}=$ 


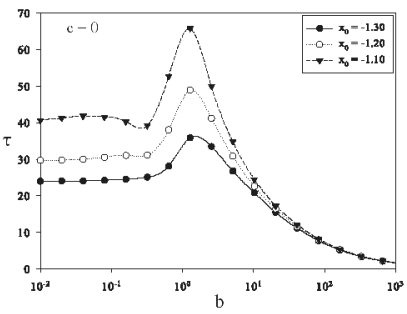

FIGURE 9. Mean escape time $\tau$ for 3 different unstable starting positions, when only the reverting term is present: $a=10^{-2}, c=0$ ).

-1.5 . When the starting position is chosen in the instability region $x_{o}<x_{M}, \tau$ exhibits an enhancement behavior, with respect to the deterministic escape time, as a function of $v$. This is the NES effect and it can be explained considering the barrier "seen" by the Brownian particle starting at the initial position $x_{0}$, that is $\Delta U_{i n}=U\left(x_{\text {max }}\right)-U\left(x_{0}\right)$. In fact $\Delta U_{i n}$ is smaller than $\Delta U$ as long as the starting position $x_{0}$ lies in the interval $I=\left[x_{I}, x_{M}\right]$. Therefore for a Brownian particle starting from an unstable initial position, from a probabilistic point of view, it is easier to enter into the well than to escape from, once the particle is entered. So a small amount of noise can increase the lifetime of the metastable state. For a detailed discussion on this point and different dynamical regimes see Refs. $[16,17]$. When the noise intensity $v$ is much greater than $\Delta U$, the Kramers behavior, expressed by the exponential law $\tau=A \exp [\Delta U / v]$ (with $v$ the noise intensity), is recovered.

Here, by considering the modified Heston model, characterized by a stochastic volatility and a nonlinear Langevin equation for the returns, we study the mean escape time as a function of the model parameters $a, b$ and $c$. In particular we investigate whether it is possible to observe some kind of nonmonotonic behavior for $\tau$. We call the enhancement of the mean escape time (MET) $\tau$, with a nonmonotonic behavior as a function of the model parameters, NES effect in the broad sense. Our modified Heston model has two limit regimes, corresponding to the cases $a=0$, with only the noise term in the equation for the volatility $v(t)$, and $c=0$ with only the reverting term in the same equation. This last case corresponds to the usual parametric constant volatility regime. In fact, apart from an exponential transient, the volatility reaches the asymptotic value $b$, and the NES effect is observable as a function of $b$. To this purpose we perform simulations by integrating numerically the equations (11) and (12).

The mean escape time as a function of $b$ is plotted in Fig. 9 for the 3 different starting unstable positions and for $c=0$. The curves are averaged over $10^{5}$ escape events. The nonmonotonic behavior is present. After the maximum, when the values of $b$ are much greater than the potential barrier height, the Kramers behavior is recovered. The nonmonotonic behavior is more evident for starting positions near the maximum of the potential. For $a=0$ the system is too noisy and the NES effect is not observable as a function of parameter $c$. The presence of the reverting term therefore affects the behavior of $\tau$ in the domain of the noise term of the volatility and it regulates the transition from nonmonotonic to monotonic regimes of MET. The results of our simulations show that the NES effect can be observed as a function of the volatility reverting level $b$, the effect 
being modulated by the parameter $(a b) / c$. The phenomenon disappears if the noise term is predominant in comparison with the reverting term. Moreover the effect is no more observable if the parameter $c$ pushes the system towards a too noisy region. When the noise term is coupled to the reverting term, we observe the NES effect on the variable $c$. The effect disappears if $b$ is so high as to saturate the system [19].

\section{CONCLUSIONS}

The enhancement of stability in systems with metastable states together with the role played by the resonant activation effect were presented in this short review. Specifically different systems were considered ranging from physics to econophysics: the Ising model, JJ devices, stochastic neuronal models, tumor-immune system, and a market model with stochastic volatility.

\section{ACKNOWLEDGMENTS}

This work was supported by MIUR and CNISM-INFM. A.F. acknowledges the Marie Curie TOK grant under the COCOS project (6th EU Framework Programme, contract No: MTKD-CT-2004-517186).

\section{REFERENCES}

1. W. Horsthemke and R. Lefever, Noise-Induced Transition, Springer-Verlag, New York, NY, 1984; L. Arnold, Random Dynamical Systems, Springer-Verlag, New York, NY, 1998.

2. J. These, The Cytokine Network and Immune Function, Oxford University Press, Oxford, UK, 2000.

3. A. Stephenson, Memoirs and Proceedings of the Manchester Literary and Philosophical Society 52, $1-10(1908)$.

4. M. San Miguel, and R. Toral, Stochastic effects in physical systems, in Instabilities and Nonequil. Structures VI, 35 - 130, E. Tirapegni and W. Zeller, eds., Kluwer, The Netherlands, 1997.

5. J. Garcia-Ojalvo and J. M. Sancho, Noise in Spatially Extended Systems, Springer- Verlag, NY 1999.

6. A. J. Legget, Phys. Rev. Lett. 53, 1096 (1984); Muthukumar, ibid. 86, 3188 (2001).

7. C. R. Doering and J. C. Gadoua, Phys. Rev. Lett. 69, 2318 (1992).

8. R. N. Mantegna and B. Spagnolo, Phys. Rev. Lett. 84, 3025 (2000); M. Bier and R. D. Astumian, ibidem 71, 1649 (1993); A. L. Pankratov and M. Salerno, Phys. Lett. A 273, 162 (2000).

9. Y. Yu and S. Han, Phys. Rev. Lett. 91, 127003 (2003).

10. A. A. Dubkov, N. V. Agudov and B. Spagnolo, Phys. Rev. E 69, 061103 (2004).

11. A. L. Pankratov and B. Spagnolo, Phys. Rev. Lett. 93, 177001 (2004).

12. E. V. Pankratova, A. V. Polovinkin, and B. Spagnolo, Physics Letters A 344, 43-50 (2005).

13. A. Fiasconaro and B. Spagnolo, A. Ochab-Marcinek and E. Gudowska-Nowak, Phys. Rev. E 74, 041904(10) (2006).

14. G. Sun et al., Phys. Rev. E 75, 021107(4) (2007).

15. B. Spagnolo et al., Acta Phys. Pol. 38, 1925 (2007).

16. N. V. Agudov and B. Spagnolo, Phys. Rev. E 64,035102(R) (2001); J. E. Hirsch et al., Phys. Rev. A 25, 519 (1982); R. N. Mantegna and B. Spagnolo, Phys. Rev. Lett. 76, 563 (1996); R. Wackerbauer, Phys. Rev. E 59, 2872 (1999); A. Mielke, Phys. Rev. Lett. 84, 818 (2000).

17. A. Fiasconaro, B. Spagnolo and S. Boccaletti, Phys. Rev. E 72, 061110(5) (2005).

18. Pablo I. Hurtado, J. Marro, and P. L. Garrido, Phys. Rev. E 74, 050101(R) (2006).

19. Giovanni Bonanno, Davide Valenti, and Bernardo Spagnolo, Phys. Rev. E 75, 016106 (2007).

20. H. A. Kramers, Physica 7, 284 (1940).

21. S. Bleil, P. Reimann, and C. Bechinger, Phys. Rev. E 75, 031117 (2007). 